\title{
Estudios Sociales
}

\section{Revista de Alimentación Contemporánea y Desarrollo Regional \\ Volumen 30, Número 56. Julio - Diciembre 2020 \\ Revista Electrónica. ISSN: 2395-9169}

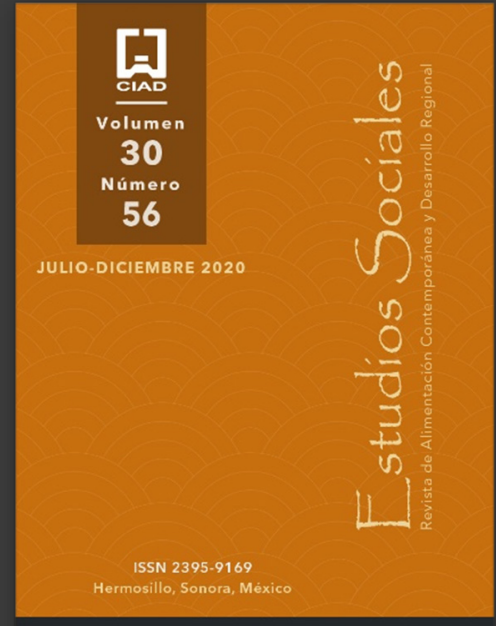

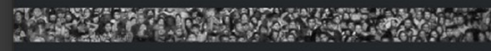

La evolución de los gustos: el consumo del café y el vino como incipiente marca de distinción social.

The evolution of tastes: coffee and wine consumption as an incipient mark of social distinction

DOI: https://dx.doi.org/10.836/es.v30i56.1000

e201000

Sergio Lorenzo Sandoval-Aragón*

http://orcid.org/0000-0003-1503-0758

Fecha de recepción: 27 de junio de 2020.

Fecha de envío a evaluación: 03 de agosto de 2020 .

Fecha de aceptación: 25 de agosto de 2020.

*Universidad de Guadalajara. Centro Universitario de La Ciénega. Centro de Estudios Sociales y Regionales (CESOR).

Departamento de Política y Sociedad.

Avenida Universidad No. 1115, Lindavista.

Biblioteca Fernando del Paso Planta Baja. C. P. 47810.

Ocotlán, Jalisco. México.

Tel. +52 (392) 925 9400, Ext. 48397

Dirección electrónica: sergio.sandoval@cuci.udg.mx

Centro de Investigación en Alimentación y Desarrollo, A. C.

Hermosillo, Sonora, México. 


\section{LA EVOLUCIÓN DE LOS GUSTOS: EL CONSUMO DEL CAFÉ Y EL VINO COMO INCIPIENTE MARCA DE DISTINCIÓN SOCIAL}

\section{Resumen / Abstract}

Objetivo: Explicar la aparición de nuevos consumos y prácticas alimentarias, específicamente del café gourmet y del vino de mesa. Metodología: Además de una revisión bibliográfica exhaustiva, se realizó la explotación sistemática de bases de datos estadísticos internacionales, nacionales y locales, así como el trabajo de campo que incluyó la encuesta, la entrevista y la observación in situ. Resultados: El ensayo muestra que el consumo del café gourmet y particularmente el vino de mesa, y las prácticas asociadas al mismo, están surgiendo o fortaleciéndose, por lo que se puede esperar que funcionen como signos de distinción social, pues sociológicamente su consumo ha sido asociado con la relativa elevación y redistribución del capital cultural y/o del capital económico. Limitaciones: Este ensayo es un primer acercamiento, el cual deberá sentar el precedente para un estudio de mayor amplitud y duración. En este sentido, es una contribución teórica y metodológica para el desarrollo de estudios más profundos, para los que un acercamiento etnográfico, más sistemático y prolongado, resultará una vía primordial. Conclusiones: Las transformaciones en los "gustos" de la población, inducidos por los cambios en el volumen y estructura del capital cultural y del capital económico, están generando cambios cuantitativos y cualitativos en el mercado de los alimentos y las bebidas, con cambios de modalidad en las relaciones al interior de, y entre, las clases sociales.
Objective: To explain the emergence of new consumption and food practices, specifically gourmet coffee and table wine. Methodology: In addition to an exhaustive bibliographic review, the systematic exploitation of international, national and local statistical databases was carried out, as well as the fieldwork that included the survey, interview and on-site observation. Results: The essay shows that the consumption of gourmet coffee and particularly table wine, and the practices associated with it, are emerging or strengthening as signs of social distinction or "social markers", since sociologically their consumption has been associated with the relative elevation and redistribution of cultural capital and / or economic capital. Limitations: This essay is a first approach, which should set the precedent for a study of greater scope and duration. In this sense, it is a theoretical and methodological contribution to the development of deeper studies, for which a more systematic and prolonged ethnographic approach will prove to be a primary route. Conclusions: The changes in the "tastes"; of the population, induced by changes in the volume and structure of cultural capital and economic capital, are generating quantitative and qualitative changes in the food and beverage market, with changes in modality in the relationships within and between social classes.
Palabras clave: alimentación contemporánea; café gourmet; vino de mesa; capital cultural; capital económico; marca social.
Key words: contemporary food; gourmet coffee; table wine; cultural capital; economic capital; social mark. 


\section{Introducción}

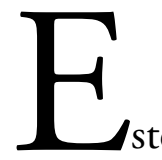
las prácticas y consumos culturales o estilos de vida y su relación con cambios de orden económico y social en una ciudad media en México. ${ }^{1}$ En ese estudio se trató de comprobar si acaso la presencia de servicios de educación superior pública en la localidad, especialmente de la Universidad de Guadalajara, ha producido cambios en el volumen y estructura de los capitales cultural y económico de la población, contribuyendo a la modificación de las estructuras sociales, particularmente las relaciones de género, la estructura de las unidades familiares, ${ }^{2}$ la dinámica poblacional, el ascenso social transgeneracional e incluso el sistema de valores, así como de las estructuras económicas, especialmente la comercialización de productos antes inexistentes o escasos y la aparición de nuevos servicios, así como la modificación del mercado de trabajo local. ${ }^{3}$ El estudio se desarrolló según un modelo teórico inspirado en la obra de Pierre Bourdieu, útil para el estudio de las prácticas alimentarias en diversos contextos nacionales y diversos momentos históricos, en virtud de su capacidad de vincular estructuralmente las dimensiones sociales, culturales y económicas. ${ }^{4}$

La investigación dio cuenta de complejas transformaciones, a partir de mediados de la década de 1990, en las estructuras sociales y económicas en esa ciudad, correlativas con cambios generales en el capital cultural y el capital económico de su población. Se determinó que la presencia de la Universidad ha contribuido de manera decisiva, pero no exclusiva, en dichas transformaciones, pues también han contribuido las nuevas tecnologías de la comunicación y el intercambio cultural 
debida a su cercanía con la capital del estado de Jalisco y al hecho de que Ocotlán posee un índice "alto" de comunicaciones y, secundariamente, a una relativa dinámica migratoria con los Estados Unidos de América (IIEG, 2018). Finalmente, la aparición de nuevas actividades industriales y comerciales a finales del siglo XX (la fabricación industrial de muebles), también ha contribuido de manera notable al auge de un mercado de los servicios alimenticios y de hospedaje.

En el contexto de ese estudio, se identificaron cambios en los consumos y prácticas alimentarias, tales como la generalización de la práctica de tomar alimentos fuera del ámbito doméstico. Se distinguen dos grupos de prácticas y consumos: el primero, tiene que ver con el "comer fuera" (Warde, 2016, p. 157-164) como parte del tiempo de ocio y convivencia, mientras que el segundo está relacionado con prácticas como estudiar o trabajar. ${ }^{5}$ Asimismo, se identificaron cambios en las relaciones de género y en la relación de las parejas con el dinero. Incluso se identificaron cambios estructurales en el "mercado matrimonial" local asociados con la valoración del papel de la mujer en la alimentación familiar (Sandoval, en prensa).

Pero el estudio también arrojó evidencias de transformaciones en los "gustos" de la población, inducidos por los cambios en el volumen y estructura del capital cultural, que no sólo están generando cambios (cuantitativos y cualitativos) en el mercado de alimentos y bebidas, sino también, cambios en las relaciones de género $y$ en las relaciones entre clases sociales mismas, toda vez que esas prácticas y consumos que están surgiendo en la población observada, pueden fortalecerse como signos de distinción social. Se analizó, de manera complementaria, la evolución reciente en el consumo de café (cafeterías gourmet) ${ }^{6}$ y de bebidas alcohólicas, particularmente el "vino de mesa", bebidas que constituyen importantes

"marcadores sociales" pues su consumo está fuertemente asociado con la relativa elevación del capital cultural y/o del capital económico en las poblaciones (Benedittis, 2019; Schwarz, 2013). ${ }^{7}$

\section{La matriz teórica de La distinción}

En la obra capital que inspira nuestro marco teórico, La distinción, Bourdieu se propuso romper con una visión económica ingenua que se limita a establecer una relación mecánica e inmediata entre el nivel de ingresos de los diferentes grupos sociales y sus prácticas y consumos culturales, sobre todo en materia alimentaria. 
Los consumos y las prácticas culturales más refinadas no se explican solamente a partir del capital económico que poseen los agentes para costearlas, sino también por el volumen de su capital cultural, los cuales se expresan socialmente como "gustos" asociados a las clases sociales (Bourdieu, 1998). ${ }^{8}$

La teoría del espacio y los campos sociales es una astucia teórica y metodológica que permite controlar cualquier preconcepción sobre "la sociedad" y asegurar la mayor objetividad posible. Comienza por postular que una sociedad puede entenderse teóricamente como un espacio social, es decir, como un complejo sistema de relaciones cuya estructura objetiva debe ser construida a partir de la investigación empírica, particularmente de la distribución de tres tipos de "recursos" o capitales: social, cultural y económico. ${ }^{9}$ Dentro de este espacio social, a su vez, pueden constituirse diversos campos, esto es, espacios o sistemas de relaciones más específicos, con más o menos autonomía, en relación con el espacio social y entre ellos, es decir, que se rigen por un sistema de reglas propio (autonomía relativa). En estos campos se instituye un determinado tipo de prácticas, creencias (illusio), lenguaje y organización que definen su estructura y distribución de los recursos (caracterizados como "capitales") y de las posiciones de los agentes. Asimismo, aunque un campo suele presentar la tendencia a conservarse (principalmente a través de mecanismos de reproducción de las estructuras de distribución de recursos y posiciones), existen diversos factores, tanto endógenos (principalmente las mismas luchas internas por definir al propio campo), como exógenos (generalmente, por la interacción con otros campos o por transformaciones en las estructuras sociales generales), que eventualmente provocan su transformación. Como una sociedad incluye una enorme complejidad y diversidad de estructuras y procesos históricos, las transformaciones pueden darse de manera localizada y diferida en el tiempo, pero en el mediano y largo plazo, dichas transformaciones alcanzan a todo el espacio social (Smelser y Swedberg, 2005).

De manera complementaria, Pierre Bourdieu introdujo en su construcción teórica el concepto de habitus, esto es, el estado incorporado (o interiorizado) de las estructuras sociales objetivas, y por consiguiente, su contraparte subjetivacorporal. ${ }^{10}$ Así, el habitus es un concepto que permite explicar cómo los agentes hacen existir la sociedad misma, dado que las formas en que actúan, piensan y perciben las han recibido de la misma sociedad. Es en y por la práctica que al mismo tiempo emergen, se reproducen y (tarde o temprano) se transforman las estructuras sociales (Bourdieu, 2001). Según Bourdieu los habitus son los diferentes sistemas de 
disposiciones que los agentes adquirieron mediante la interiorización (o "incorporación") de un tipo determinado de condiciones sociales y económicas y que encuentran, en una trayectoria definida dentro de un campo, una oportunidad para actualizarse. Así, los "gustos" alimentarios (entre otros), no son sino la expresión de unos habitus históricamente constituidos, según determinadas condiciones y posiciones sociales, y particularmente según la relación de los agentes con determinados campos. Como afirman Melendez y Cañez,

los gustos, los significados, los valores, las preferencias y las percepciones que se relacionan con nuestras prácticas alimentarias y culinarias han sido estructurados -y son a su vez elementos estructurantes- en este sistema (...) que constituye el habitus que hemos interiorizado en el transcurso de nuestras vidas (Melendez y Cañez, 2010, p. 193).

\section{Caso 1. El café gourmet y los factores del refinamiento de los gustos}

Un acto aparentemente insignificante como lo es el consumo del café, como bien ha observado el sociólogo británico Anthony Giddens, es en sí mismo lo que podríamos llamar, con Marcel Mauss, un "hecho social total": ${ }^{11}$ es, al mismo tiempo, un ritual con una carga simbólica y un acto que remite a una estructura económica (los mercados globalizados), lo mismo que a una compleja historia social y política que va desde las relaciones de explotación coloniales, hasta la actual defensa del medio ambiente (Giddens, 2017). Pero, desde una visión de la realidad menos "encantada" que la sostenida por Giddens, el consumo del café también hace las veces de un marcador de distinción social (Roseberry, 1996). ${ }^{12}$

Según el CEDRSSA (Centro de Estudios para el Desarrollo Rural Sustentable y la Soberanía Alimentaria), aunque el café en grano se ha cultivado y consumido desde finales del siglo XVIII (CEDRSSA, 2018: 3), en México existe desde hace décadas un extenso consumo de café soluble. El café soluble fue patentado y comercializado por Nestlé en 1938 (NESTLÉ, 2020) y fue introducido en México casi de inmediato. ${ }^{13}$ Sin embargo, su consumo se vio incrementado espectacularmente en las décadas de 1970 y 1980 gracias a la intensa publicidad televisiva (CAFIVER, 2020) y es consumido principalmente en los hogares, sobre todo de clase popular, pues "la desigualdad económica sigue determinando el perfil del consumidor” (Euromonitor; 2017: 31). 
Actualmente, empero, el consumo del café en grano, que principalmente se consume en cafeterías, esto es, fuera de casa, se ha venido incrementando y en 2016 se calculó que aproximadamente el $80 \%$ del café vendido en restaurantes y cafeterías era molido y en grano (Euromonitor, 2017: 35). En conclusión, el incremento en el consumo del café natural está asociado a dos rasgos importantes de sus consumidores: son principalmente con ingresos altos y jóvenes (Euromonitor, 2017: 39 y 56). Aunque los estudios revisados no hacen referencia al nivel de escolaridad, sabemos que el nivel de ingresos está correlacionado con el nivel de escolaridad (Favila, 2017), por lo que se puede asumir que la escolaridad elevada (en todo caso, superior a la media) es un rasgo adicional del perfil del consumidor.

Como se mencionó, diversas transformaciones sociales, culturales y económicas en la población observada en los últimos 25 ańos, han favorecido un incremento importante de la venta de alimentos preparados, entre ellos la de bebidas a base de café gourmet. ${ }^{14}$ Sin embargo, a diferencia de lo que pudiera pensarse, no podemos contentarnos solo con las correlaciones estadísticas que nos dan evidencias de que ha habido cambios e, incluso, de sus causas, pero muy poco acerca de cómo se han dado esos cambios. ${ }^{15} \mathrm{~A}$ la pregunta sobre cuáles factores influyen en ese cambio, le sigue inevitablemente otra: ¿Cómo y en qué momento se produce un cambio en el gusto de los individuos? No es posible aquí agotar este tema, pero sí podemos adelantar algunos indicios. ${ }^{16}$

Los "gustos" dependen ciertamente de los bienes y servicios disponibles en un lugar y un momento dados (Meléndez y Cañez, 2010, p. 185). Pero también, si las condiciones de existencia de la población y de los individuos cambian, eso genera cambios en los gustos, que a su vez modifican los bienes y servicios disponibles:

los gustos efectivamente realizados dependen del estado del sistema de los bienes ofrecidos, ocasionando cualquier cambio del sistema de los bienes un cambio de los gustos; pero a la inversa, todo cambio de los gustos resultante de una transformación de las condiciones de existencia y de las correlativas disposiciones conduce a determinar, más o menos directamente, una transformación del campo de producción (Bourdieu, 1998, p. 271).

Considérese, por ejemplo, el siguiente pasaje de una entrevista a un joven empresario, dueño de cafeterías llamadas "de concepto". El entrevistado nació en Guadalajara, Jalisco, pero creció en la población de Tototlán, ${ }^{17}$ que colinda con Ocotlán. Hijo de una familia de comerciantes, estudió la carrera de mercadotecnia 
en una universidad privada en Guadalajara, lo cual indica una condición económica relativamente favorable. Obtuvo su cédula profesional en 2006. Al preguntarle cómo fue que decidió iniciar ese negocio, respondió:

Primero [fue] la idea era un bar, pero la situación en Ocotlán no estaba para nada bien, entonces decidí suspenderla por seguridad. En septiembre de 2012, hubo una exposición de café en ciudad de México, la más grande de toda Latinoamérica. Fui, me gustó, me capacité y cuando venía de Ciudad de México, yo ya venía con la idea de un proyecto sobre café. Yo no tomaba café, pero como que le agarré cierto cariño, primero al aroma y después al hecho de estarlo trabajando, le agarré el gusto al café. Pero de ahí surge, de una exposición (entrevista dueño de cafeterías, 35 años).

Como puede observarse, el entrevistado dice que no acostumbraba tomar café antes de haberse informado sobre la oportunidad de negocio que significaba una cafetería en 2012. Es decir, desarrolló el gusto por el café ya siendo un treintañero. Pero ¿qué hubo antes? En breve: una intensa exposición a la vida urbana, estudios universitarios, la experiencia no fortuita de una "expo" sobre la industria del café en el World Trade Center México y, finalmente, su ingreso en el negocio del café. En resumen, lo que produce o eventualmente transforma un "gusto" es la relación entre las estructuras objetivas (económicas y sociales) y las estructuras subjetivas (habitus). En el caso de la población que fue objeto de estudio, la presencia de la Universidad de Guadalajara ha sido un factor decisivo. Así, al preguntar a nuestro entrevistado cómo fue que decidió mover su negocio de cafetería del centro de la ciudad a las cercanías de la universidad y, finalmente, al interior de la misma por medio de una concesión, respondió:

Nos cambiamos hacia la Universidad: [la clientela] cambia totalmente, casi un $80 \%$ son estudiantes [...] Aquí es una zona muy comercial porque hay mucho estudiante y parte de la gente que teníamos en el Centro eran estudiantes y de repente nos [preguntaban] que por qué no habríamos algo más cerca. Comenzamos a realizar los estudios de mercado y resultó que muchas de las personas que teníamos todos los días de la semana eran estudiantes y la mayoría de aquí de la U de G. El hecho de venirnos fue para atender esa demanda que nos estaban pidiendo (entrevista dueño de cafeterías, 35 años). ${ }^{18}$ 
En definitiva, la presencia de la universidad en esta población no solo ha contribuido a aumentar el mercado de la preparación y venta de alimentos, sino que también lo ha diversificado, como afirma nuestro entrevistado:

¿Consideras que han cambiado los tipos de establecimientos de alimentos y bebidas y como ha sido ese cambio?

- Sí han cambiado, no se podría decir que, a través de nosotros, pero cuando iniciamos [...] no existía una cafetería con un concepto como tal. Cuando iniciamos nosotros en 2012, iniciamos con una cafetería que se llamaba "cafetería de concepto", en dónde se creaba una atmósfera o un ambiente muy distinto al que estaban acostumbrados, en el cual se llegaba a la cafetería y sentías una sensación de que estabas en otro lugar. A partir de esa fecha se comenzó a notar el cambio en Ocotlán en cuanto a los bares, restaurantes. Ahora cerca de la universidad se han abierto varios bares con conceptos muy diferentes. Sí han cambiado, muchísimo.

¿En ese tiempo de 2012 si existian muchas cafeterias?

- Inicialmente, cuando nosotros entramos creo que había dos más en Ocotlán, nosotros fuimos la tercera marca y a partir de eso ya se abrieron más lugares de café (entrevista dueño de cafeterías, 35 años).

La aparición de este tipo de cafeterías en esta localidad, en resumen, obedece a las transformaciones económicas y culturales de las últimas décadas. Pero también la adquisición del gusto por el café, cuyo "paradigma" sería el estilo espresso (Morris, 2013), tanto en el caso de nuestro entrevistado como en el de su clientela, parece obedecer, en realidad, a un largo y bien conocido periplo histórico ${ }^{19}$ que inicia con su recolección o producción en un lugar geográfico restringido, pasando por su consumo como producto suntuario, hasta su eventual abaratamiento y consumo generalizado (doméstico y rutinario), llegando, incluso, a hacer las veces de proletarian hunger killer ("mata-hambre proletario") ${ }^{20}$ y desembocando, actualmente, en una bebida preferida por un segmento de la sociedad "no tanto burgués como cortés, gentil, cosmopolita" identificada con el yuppie: ${ }^{21}$ 
Se podría ver que representa un intento de recrear, a través del consumo, un tiempo antes de la sociedad de masas y el consumo de masas. Podría verse, entonces, como una inversión simbólica de las fuerzas económicas y políticas a través de las cuales surgió este segmento de clase en particular (Roseberry, 1996: 774).

El café, nos informan múltiples estudios, funciona como un "marcador social", tanto en sociedades metropolitanas como en sociedades surgidas de dominaciones coloniales (como México). Por ejemplo, así ha sido analizado en India, donde "mientras que el café se convirtió en el marcador cultural de la clase media tamil (hindú), el té fue visto como una bebida de la clase trabajadora y/o musulmana" (Venkatachalapathy, 2002, p. 316). Puesto que el consumo del café aporta importantes claves para entender los procesos y las estructuras sociales, puede ser objeto de estudio sociológico y su función de "marca social" se manifiesta incluso en los estudios sobre las "preferencias" de los consumidores realizados en función de sus propiedades intrínsecas (organolépticas) y técnicas de preparación para su consumo (ver p. ej. Spence y Carvalho, 2019). ${ }^{22}$ No presenciamos, pues, otra cosa que el fenómeno que hace 25 años ya vislumbraba William Roseberry, cuando afirmaba de las nuevas cafeterías que:

Su éxito continuo dependerá de los procesos de diferenciación social y cultural que marquen, incluso cuando las ubicaciones sociales de los grupos de consumidores sean borrosas. También dependerá de la existencia continuada, en el país y en el extranjero, de un mundo de relaciones de explotación, evidenciado en las relaciones sociales a través de las cuales se produce el café, el compromiso y la desconexión de las regiones productoras de café en condiciones de libre mercado y los procesos de estandarización y concentración a las que se ha sometido la producción y comercialización de café gourmet (Roseberry, 1996, p. 774).

En efecto, en México, la globalización, junto con la pauperización campesina han causado un relativo abandono de la producción de café, mientras que paradójicamente proliferan las cadenas de cafeterías y los cafés extranjeros (Griffith, Zamudio, Cortés y Cabrera, 2017). Ante este preocupante panorama, solo resta preguntarnos si acaso el incremento de los capitales económico y cultural, correlativos a los procesos de urbanización y gentrificación no posibilitarán también, eventualmente, una crítica y un activismo social que lleguen a contrarrestarlo. 


\section{Caso 2. Una incipiente cultura del vino}

Bebida milenaria, el vino (fundamentalmente, el vino tinto), no solo está simbólicamente asociada a ritos y ceremonias, desde las bacanales, pasando por los ritos religiosos (por ejemplo, en el catolicismo), hasta las celebraciones más mundanas e íntimas, sino también a las estructuras sociales en sus diferentes niveles. $^{23}$

Comparado con otros países, en México, el consumo de vino es muy bajo, solo un $17 \%$ consumen vino, esto es 0.6 litros per cápita y "no existe una cultura de beberlo a diario" (Gennari y Estrella, 2015: 13). Sin embargo, se registra un incremento en el consumo de vinos. Después de Brasil, México es el segundo importador de vinos más importante de América Latina y en las últimas décadas ha incrementado su producción y exportación de vinos (Gennari y Estrella, 2015: 3). Mientras que en la última década la tendencia mundial en el consumo de vino iba a la baja (Medina, Martínez y Ramon, 2014), en México aumentó. ${ }^{24}$ Sin embargo, estudios recientes muestran que la crisis del COVID-19 de 2020 afectará a la industria vitivinícola. ${ }^{25}$

En comparación con otras bebidas alcohólicas el vino, en tanto bebida "generosa" (es decir, moderado grado alcohólico) y en tanto bebida más exigente en cuanto a su degustación, ${ }^{26}$ representa una opción al mismo tiempo más costosa. Lo cual es coherente con el hecho de que el consumidor promedio de vinos posee capitales económico y cultural mayor al promedio; es decir: tiende a parecerse a una élite. Así lo evidencia un estudio realizado durante el 2008:

el consumidor de vinos mexicano posee un alto nivel académico, buenas condiciones económicas (ingresos medios/altos) y preferencia por marcas importadas. Han comenzado a involucrarse en el tema vitivinícola, su historia y procesos; participando de clubes de vinos, realizando cursos y degustaciones. Los consumen con mayor frecuencia y eligen al momento de acompañar sus comidas o reuniones (Gennari y Estrella: 2015: 21). 
De manera más general, se sabe que en México

los consumidores de vino tienen alto grado de formación: graduados universitarios (58\%), con educación secundaria (29\%), estudiantes (7\%), técnicos (4\%) y otros (2\%). Laboralmente un $55 \%$ son empleados y en menor medida se dedican a actividades como el comercio (7\%), estudiar ( $4 \%)$, amas de casa (3 $\%$ ), jubilados o retirados (2\%) (Gennari y Estrella, 2015. p. 21).

Entre los consumidores se distinguen dos segmentos: el primero, y más importante, es la "tradicional clase alta" (Traditonal Upper Class) y el segundo son jóvenes (young tigers), hombres y mujeres entre 25 y 40 años, bien educados (formación profesional), trabajan para empresas transnacionales o grandes empresas nacionales, recién se inician en la cultura del vino y son buenos compradores de vinos (early adopters, high buyers) (Vinísfera, s.f.: 13). En conclusión, hoy en México, el vino es un producto consumido en lo general por una minoría "educada" y "con buenos ingresos", es decir, que constituye un consumo de lujo y, por consiguiente, tiene una "marca social" (Cardon y García, 2012, p. 2).

Si en México el consumo de vinos de mesa en una escala significativa es reciente, en Ocotlán lo es aún más. ${ }^{27}$ En este respecto, para el caso específico de Ocotlán no ha sido posible contar con estadísticas oficiales precisas. El Ayuntamiento solo reporta rubros muy generales, especialmente el de "venta de vinos y licores en botella cerrada" y los datos disponibles solo muestran que el número se ha mantenido en torno a una cincuentena de establecimientos. Por lo que recurrimos a un acercamiento más empírico. ${ }^{28}$

Tanto los restaurantes más antiguos, así como los establecimientos de venta, han ido introduciendo los vinos de mesa en sus menús y existencias conforme la demanda se ha incrementado en los últimos lustros, diversificando paulatinamente la variedad de marcas (y precios). Algunos restaurantes establecidos más recientemente han ofrecido vinos como parte de sus menús, como es el caso de un restaurante tipo italiano u otro de tipo espańol. Finalmente, en 2017 se abrió en Ocotlán un establecimiento en el que los vinos de mesa son la oferta principal. Este último establecimiento, junto con los recientes restaurantes de corte europeo, ya no solo reaccionan a una demanda directa, sino a una demanda potencial (presumiblemente, aquel segmento "educado y con buenos ingresos") ${ }^{29}$ y comienzan a difundir la cultura del vino. Como reza la información en su página publicitaria: 
Comprometidos con darte la mejor experiencia, en el maridaje y consumo de la bebida que, por excelencia, se ha considerado como la mejor ¡durante siglos! (sic). Considerado bebida de dioses, saludable e incluso un alimento, el consumo del VINO TINTO es un ARTE, que te ayudaremos a apreciar y atesorar (itálicas nuestras). ${ }^{30}$

En el otro extremo del polo comercial, al que corresponde el otro polo del espectro social, encontramos a los viejos comerciantes de licores, aquellos que sobreviven de un estado anterior del espacio social y del campo comercial y que ahora proveen principalmente a las clases populares y a estudiantes con poco dinero y muchas ganas de fiesta. Entre esos comerciantes elegimos a uno particularmente interesante, por su antigüedad, su popularidad y por encontrarse en pleno centro de la ciudad. En suma: por ser vestigio de una forma de comercio tradicional casi desaparecida.

Don Ramón, de 80 ańos de edad, es propietario de un negocio de venta de bebidas alcohólicas, conocido, según reza un viejo anuncio en la verde fachada, como "Vinos finos de Ocotlán", ${ }^{11}$ anuncio luminoso de metal y acrílico que da testimonio de tiempos de algún esplendor pasado. En su juventud don Ramón fue empleado en la fábrica de Celanese durante veinte años. En 1987, cuando dejó su empleo y se pensionó, compró el negocio a su primer propietario quien lo abrió en 1955. Como él mismo nos confirmó, entre risas, es conocido popularmente como "don Ilegal" por la conseja de que suele vender licor fuera de los horarios permitidos.

Nos recibe en su local, la sala de recepción de una antigua casa de estilo colonial sin duda centenaria, la cual ha sido modificada para que haga las veces de un comercio. Mercancías apiladas por doquier, en su mayoría cajas de cervezas, dejan poco espacio para recibirnos, toda vez que los clientes no acceden al local, sino que son atendidos, con ayuda de su hija, a través de las rejas que lo separan del exterior. Viejas estanterías de madera que rodean todo el espacio útil de dos paredes, soportan una variedad de empolvadas botellas de licores, predominando los tequilas. No faltan, por aquí y por allá, antiguos vestigios de publicidad que llaman a la nostalgia. En este ambiente un tanto caótico y sombrío, casi "gótico", que haría las delicias de cualquier brocanteur, sostenemos nuestra conversación. Don Ramón nos informa que, aunque la cerveza es lo que más vende, los destilados son su segundo producto más vendido. Su experiencia como comerciante durante más de treinta años nos permite reconstruir rápidamente la evolución del consumo de bebidas alcohólicas en Ocotlán: 
Cuando yo llegué aquí hace 30 años se vendía mucho el Don Pedro, el Presidente [ambas marcas populares de brandy]. Para que se dé una idea: yo llegué a tener hasta 30 cajas de Presidente y de Don Pedro diez o quince cajas ${ }^{32}$ [...] Entonces, ahorita tengo una caja de Presidente, Don Pedro también, porque... ya no se vende igual. Los tequilas superaron a los brandys. El tequila se vendía muy poco cuando yo llegué aquí, el tequila se vendía poco.

- ¿Habia muchas marcas de tequila entonces?

No había tantas como ahora, pero mejoraron mucho. Entonces la gente está cambiando. Ahorita lo que se vende hasta la fecha es más el tequila que el brandy, en esta región. Y marcas, pues han desaparecido muchas, porque algunas bajan de venta, porque llegan otras y las opacan... por el precio, la calidad.

En efecto, el relato de nuestro entrevistado reproduce, a escala, la historia del ascenso de la industria del tequila en México y en el mundo. Versión industrializada del originario mezcal, ${ }^{33}$ el "tequila" (llamado así por una polémica denominación de origen) se convirtió en las últimas décadas en el principal rival de los destilados otrora dominantes en el mercado, sobre todo debido a que las principales marcas han pasado a ser propiedad de grandes emporios transnacionales. De manera que el relato de este entrevistado refleja fielmente la historia de este sector del campo económico, demostrando que lo global no puede ser comprendido más que localmente (García, 2018).

Mientras que el tequila, bebida de origen popular y rural, lo mismo que el mezcal en general más recientemente, logró posicionarse en el ámbito comercial a través de un determinado proceso de ennoblecimiento a través de una "relegitimización por lo internacional" (García, 2018, p. 194-205), los vinos de mesa han de pasar por un proceso inverso: a diferencia del tequila y del mezcal, que se "ennoblecieron" por la calidad social de sus consumidores "nobles" (personajes famosos y connaisseurs extranjeros), el vino en cambio ennoblece a sus consumidores. Históricamente, se trata de un proceso en el que bebidas y alimentos, lo mismo que muchas prácticas "culturales", sufren un ennoblecimiento o (re)legitimización cuando son adoptados por los estratos dominantes, a partir de lo que se convierten en signos de distinción. En esos casos, se establece una barrera económica (precios elevados) que los convierten en "bienes raros" y limitan así a las clases dominadas (populares principalmente) el acceso a ellos. 
Ciertamente, como sucede con el mercado de la ropa, existe toda una gama de productos de calidad diferenciada, destinados a los diferentes estratos sociales y que refuerza la dominación simbólica en la que fundamenta su legitimación. Asimismo, en muchas sociedades se puede apreciar una redistribución social de los consumos y prácticas, pero, como bien apunta García: "El consumo de tipo 'omnívoro' se traduciría en formas más sutiles de distinción" y no en una mayor igualdad o "democratización" como se ha llegado a plantear (García, 2018, p. 196). Así, la clientela principal de nuestro entrevistado pertenece a las clases populares, de bajos ingresos y, correlativamente según nuestro modelo analítico, tiende a dejar fuera de su percepción e interés la oferta de vinos de mesa:

El vino de mesa diario se ha vendido. Pero hay negocios, como yo aquí... este me lo buscan mucho [nos muestra una botella de "tequila" de dudosa calidad]: 73 pesos el litro! [...] Mire, los vinos de mesa, aquí conmigo se venden poco realmente, yo por ejemplo nada más tengo ese [señala hacia donde hay unas cuantas botellas de Riunite... ${ }^{34}$

- Y de los vinos, ¿usted sabe [...] de vinos de mesa?

Mucho no, mucho no.

- ¿No lo vende mucho, no se lo piden mucho?

No, no lo piden [...] Con el tiempo un vino blanco se puso amarillo, con el tiempo, por eso no lo vendemos, porque tienen su tiempo [...] y es mucha pérdida, yo por eso no los vendo (Propietario de vinatería, 80 años).

En resumen: la existencia de una paulatina oferta de vinos de mesa, hasta llegar a la aparición de un establecimiento especializado, contrasta, pero se complementa, con la existencia de una nutrida oferta de bebidas más estándar y de fácil acceso (sobre todo cervezas industriales y marcas de licores de baja calidad), lo cual indica una diferenciación de los consumidores no solo en términos económicos, sino también y quizá principalmente, en términos culturales (y por lo tanto sociales). ${ }^{35} \mathrm{Y}$ la relativa elevación de la escolaridad en la población, sobre todo de tipo superior, conjuntamente con una capacidad de compra, cumple aquí un papel preponderante. ${ }^{36}$ 


\section{Conclusiones}

Según estudios sociológicos realizados en países diferentes a México, los cambios en los estilos de vida y los gustos se corresponden con cambios homólogos en la estructura de las relaciones sociales (relaciones "horizontales" como pueden ser las relaciones entre hombres y mujeres, o relaciones "verticales" entre estratos o clases), así como también con cambios en los mercados de productos y bienes entre ellos, los alimentarios, por lo que los consumos alimentarios, y las prácticas asociados a ellos, suelen funcionar también como marcas de distinción social.

La investigación realizada en una ciudad media del occidente del país (Ocotlán, Jalisco), arrojó evidencias de que, efectivamente, los cambios en el volumen y estructura del capital cultural y del capital económico corresponden a transformaciones en los "gustos" de la población, generando a su vez cambios cuantitativos y cualitativos en el mercado de los alimentos y las bebidas. Se analizaron dos casos de bebidas que han iniciado un auge económico en la población en estudio, el café gourmet y el vino de mesa.

En el caso del primero, el café gourmet, se pudo observar que la población elegida para el estudio se ha insertado, aunque de manera periférica, en un proceso histórico, descrito de manera muy precisa en otros contextos nacionales, de transformación del consumo del café asociado a ciertos grupos (o clases) sociales, especialmente los segmentos más jóvenes y con creciente nivel educativo. En el caso del segundo, el vino de mesa, se pudo dar cuenta, a través del análisis de la reestructuración del mercado de bebidas alcohólicas local, de una incipiente cultura del vino, igualmente asociada a las transformaciones recientes en el volumen y estructura del capital cultural y económico de la población. Estos resultados permiten, en consecuencia, describir una incipiente constitución de esos consumos y prácticas como marcas de distinción social que tres décadas antes no existían y que se corresponden con los cambios sociales y económicos experimentados en ese lapso.

Finalmente, se debe advertir que el trabajo aquí reportado solamente es un primer acercamiento que deberá sentar el precedente para un estudio de mayor amplitud y duración, toda vez que este tipo de estudios prácticamente son muy escasos en México. En este sentido, el presente ensayo es una contribución para el desarrollo de estudios más profundos para los que un acercamiento etnográfico, más sistemático y prolongado, además del análisis de datos estadísticos primarios, será fundamental. 
Notas al pie:

${ }^{1}$ El estudio se titula "Transformaciones en el espacio de los consumos y prácticas culturales en Ocotlán, Jalisco, y su impacto social y económico en relación con la presencia de IES en la región. Consumos y prácticas alimentarias", desarrollado en el seno del Centro de Estudios Sociales y Regionales (CESOR). Se realizó a través de la explotación sistemática de bases de datos estadísticos, así como del trabajo de campo propio, que incluyó la encuesta, la entrevista y la observación in situ. El estudio tiene como antecedente el trabajo de: Sandoval (2012) y Hernández y Calonge (2012).

2 El estudio coincidió, en la escala local, con las descripciones elaboradas en el nivel nacional por Aguilar (2017).

${ }^{3}$ El municipio de Ocotlán se encuentra en la región Ciénega de Jalisco (México), a 84 kilómetros al sureste de la capital (Zona Metropolitana de Guadalajara). Comprende 51 localidades habitadas, la principal es la cabecera municipal, del mismo nombre (Ocotlán). Pertenece a la gran cuenca Lerma-Chapala-Santiago. Villa fundada durante la Colonia, adquirió la denominación de "ciudad" en 1963.

${ }^{4}$ Aunque se ha afirmado que en México este tipo de estudios no se han desarrollado suficientemente (Bustamante y García, 2015), para el caso particular de la alimentación sí existen algunos trabajos destacables: sobre los cambios alimentarios y los estilos de vida, en relación con la cocina tradicional regional, véase: Meléndez y Cañez (2010). Sobre el significado de los alimentos como diferenciadores sociales (y de género) en un poblado rural del México contemporáneo, véase el estudio etnográfico de Ochoa (2013). Véase también el estudio sobre la percepción y los motivos de consumo del amaranto en México de Rojas, Espinoza, Thomé, Moctezuma y Cuffiac (2019), quiénes siguiendo a Bourdieu, recurren al análisis de correspondencias múltiples.

${ }^{5}$ Como ha observado Thamer, la evolución de los gustos alimentarios no solo se debe al proceso de diferenciación social, sino que también "tiene que ver con la constitución simbólica del tiempo y de las actividades" (Thamer, 2016: 78).

${ }^{6}$ Se ha optado por el término "gourmet", pues en realidad desde el punto de vista sociológico aún no existe uno mejor. Una alternativa prometedora es la de "café de especialidad", propuesta por Bravo y Villagra (2020).

${ }^{7}$ El consumo doméstico de café espresso y la cultura del vino, aparecen explícitamente como variables en un estudio realizado en Noruega y utilizando los análisis estadísticos refinados (Flemmen, Jarness y Rosenlund, 2019), demostrando la tesis de Bourdieu sobre la homología existente entre el espacio de los gustos y el espacio social.

${ }^{8}$ Curiosamente, el tema propiamente alimentario no figura en uno de los libros más recientes y más importantes sobre la actualidad internacional de la sociología de Pierre Bourdieu (Medvetz y Sallaz, 2018).

${ }^{9}$ Formas de capital que, cuando sirven para justificar alguna forma de dominación, funcionan como capital simbólico. 


\section{LA EVOLUCIÓN DE LOS GUSTOS: EL CONSUMO DEL CAFÉ Y EL VINO COMO INCIPIENTE MARCA DE DISTINCIÓN SOCIAL

10 Siendo el habitus "producto de las experiencias pasadas y de toda una acumulación colectiva e individual" el cual "solo puede comprenderse de manera adecuada mediante un análisis genético que se aplique a la vez a la historia colectiva [...] y a la historia individual" lo permite "el análisis de las condiciones económicas y sociales de la génesis de los gustos individuales en materia de alimentación, decoración, vestimenta y también canciones, teatro, música o cine, etc. y, en términos más generales, de las disposiciones [...] a llevar a cabo las acciones económicas ajustadas a un orden económico" (Bourdieu, 2001, p. 239-240).

${ }^{11}$ Un "hecho social total" es aquel que involucra a "la totalidad de la sociedad y de sus instituciones" pues "todos esos fenómenos son a la vez jurídicos, económicos, religiosos e incluso estéticos, morfológicos, etc.” (Mauss, 1923-1924, p. 102, traducción propia).

12 La similitud de los estudios sociológicos con los actuales estudios de marketing no es casual, pues estos se han beneficiado de hecho de los primeros, lo cual suele pasar desapercibido. Al respecto, ver: Moingeon (1993).

${ }^{13}$ La aparición de este tipo de cafeterías en esta localidad resulta, aún más significativo desde que se toma en cuenta que el café "instantáneo" se ha consumido ahí de manera importante, debido a la presencia de una de sus principales productoras mundiales: la planta de la legendaria empresa suiza Nestlé, se estableció en Ocotlán en 1935 siendo la primera en México dedicada a la fabricación de productos de nutrición infantil derivados de lácteos (producto originario de la empresa). En octubre de 2016 abrió en el mismo municipio una segunda planta.

${ }^{14}$ Según datos de la oficina municipal de Padrón y Licencias, entre 2005 y 2015 los establecimientos en general se habían incrementado en un $57 \%$. Una consulta en la base de datos "SIMBAD" del INEGI permitió comparar y verificar este dato.

${ }^{15}$ Por ahora, una idea debe ser retenida: aunque los gustos se transformen, no dejan de funcionar como formas de distinción social (Tissot, 2013).

${ }^{16}$ Ello requeriría de la realización de un gran número de entrevistas y observaciones etnográficas y, por lo tanto, de financiamiento y de tiempo.

17 Pequeña población que, hasta el censo de 2010, tenía 21,871 habitantes. Sus principales actividades económicas son agricultura y ganadería.

${ }^{18}$ Con una marca de género también: como un complemento del trabajo de campo, se realizó un sondeo (encuesta) aplicada entre febrero y mayo de 2019 a una muestra de estudiantes de tres carreras (Abogado, Administración e Ingeniería Industrial, $\mathrm{N}=155,52 \%$ mujeres), representativas de cada una de las tres Divisiones académicas del campus regional de la Universidad de Guadalajara en la localidad (error de muestreo de $7.61 \%$ e intervalo de confianza de $95 \%$ ). Se observó que los hombres frecuentan más las cafeterías (de los que van cuatro veces o más a la semana, el $57 \%$ son hombres), principalmente estudiantes de Ingeniería Industrial, la carrera más masculinizada.

19 "El efecto acumulativo (...) fue que el consumo de café adquirió poder expresivo como una demostración de capital cultural” (Morris, 2013, p. 897).

${ }^{20}$ Periplo similar al experimentado por el azúcar, como hace notar Bourdieu a partir de los estudios de Mintz (1996), quien ilustró "cómo la afición por el azúcar, en un primer momento producto de lujo exótico reservado a las clases privilegiadas, se convirtió poco a poco en un 
elemento indispensable de la alimentación corriente de las clases populares" (Bourdieu, 2001, p. 240).

${ }^{21}$ El Cambridge Dictionary define al yuppie como "un joven que vive en una ciudad, gana mucho dinero y lo gasta haciendo cosas de moda y comprando posesiones caras".

${ }^{22}$ El estudio ulterior de este objeto sociológico, puede beneficiarse en mucho del modelo desarrollado por el sociólogo Richard A. Peterson, el cual, según Jonathan Morris, "analiza seis facetas de la producción: tecnología, ley y regulación, estructura de la industria, estructura de la organización, carreras profesionales y percepciones del mercado por parte de los productores, en relación entre sí" (Morris, 2013, p. 882).

${ }^{23}$ Así, por ejemplo, el antropólogo Alain Testart (2014) analizó las raíces simbólicas de la costumbre, muy difundida en Europa y exportada a otras naciones, que "prohíbe" a las mujeres descorchar las botellas de vino, y también el corte y servicio de la carne, dejando esas actividades en manos de los hombres, prohibición (tabú) que actualmente se ha transformado en una refinada regla de urbanidad. Por otro lado, en México, en 2015 el 64\% de los consumidores de vino eran varones (Vinísfera, s.f.: 28).

${ }^{24}$ Según el Consejo Mexicano Vitivinícola "el nivel de participación del mercado nacional del vino ha crecido, con lo que los vinos producidos en el país tienen 30 por ciento del mercado, frente al 70 por ciento que viene de importación" aunque "la ingesta per cápita de la bebida en el país es de apenas 1 litro al ańo frente a naciones europeas donde el consumo alcanza el litro por semana" (Consejo Mexicano Vitivinícola, 2020).

25 "Ahora se espera que las ventas minoristas de vino aumenten solo un $3 \%$ en términos de volumen en general en 2020, a la luz del impacto de COVID-19. Este es un contraste moderado con el aumento del 7 \% en las ventas pronosticado en la edición 2019 de bebidas alcohólicas" (Euromonitor International, 2020).

${ }^{26}$ Así lo pone de manifiesto una guía para el consumidor, que incluye un estudio comparativo, publicado en 2015 (PROFECO, 2015).

27 Personalmente, recuerdo haber buscado en sus "vinaterías" céntricas una botella de vino tinto a mediados de los años 1990, sin éxito.

${ }^{28}$ Observación directa y conversaciones breves e informales con propietarios y dependientes, y una entrevista más detallada.

29 "Para conocer los hábitos de consumo de los vinos de mesa, entre el 28 de octubre y el 25 de noviembre del presente año [2008], la Dirección General de Estudios sobre Consumo de PROFECO aplicó un sondeo en su sitio de Internet. De un total de 317 respuestas de 29 estados de la República Mexicana los principales resultados son los siguientes: Compra mensualmente de 1 a 4 botellas. Realiza un gasto de hasta 500 pesos. Y prefiere el vino tinto" (Fuente: Brújula de compra de PROFECO, 2008).

30 Asimismo, precisan: "Somos una tienda de licores con venta de botellas en mayoreo y menudeo, y que somos capaces de conseguir cualquier tipo de botella en el mundo". Recuperado de https://www.facebook.com/pg/Vinos500lunas/posts/

31 En Jalisco, al menos, tradicional y popularmente se utiliza el adjetivo "vino" para referirse de manera general a cualquier bebida alcohólica, especialmente destilados. De ahí que se use la expresión "vino de mesa" para precisar. 


\section{LA EVOLUCIÓN DE LOS GUSTOS: EL CONSUMO DEL CAFÉ Y EL VINO \\ COMO INCIPIENTE MARCA DE DISTINCIÓN SOCIAL}

SANDOVAL-ARAGÓN

32 Cada una de esas cajas contenía doce botellas.

33 Destilado popular obtenido tradicionalmente a partir de diversas especies de agaves.

34 Vino tinto joven, espumoso, afrutado y dulce, de origen italiano, elaborado con uva de la variedad lambrusco y que se consume frío. Generalmente, hace las veces de cóctel o aperitivo.

35 Según la Encuesta Nacional de Ingresos y Gastos de los Hogares (ENIGH) 2018, el 35.3 \% del gasto de los mexicanos se dedica a "alimentos, bebidas y tabaco", representando el segundo rubro de gasto corriente monetario promedio trimestral para los veinte principales rubros específicos del gasto, siendo el primero "educación”. Jalisco ocupó en 2018 el cuarto lugar entre los cinco estados con mayor gasto corriente (INEGI, 2018).

${ }^{36}$ En cuanto a consumo de bebidas alcohólicas, nuestro sondeo mostró que los vinos de mesa no están entre las bebidas preferidas de los estudiantes. En cambio, los tequilas o mezcales, la cerveza y los whiskies (en ese orden) son las bebidas preferidas de todos, pero en los semestres más altos $\left(8^{\circ}\right)$ comienzan a diversificarse los gustos e incluyen vodkas y ginebras, brandies y cońacs, aguardientes (incluso el cachaza) y, en medida ínfima, los vinos (de todo tipo). Esto indica que el gusto por los vinos, en efecto, se desarrolla muy lentamente, junto con el capital cultural y, en su momento, el capital económico al insertarse en los mercados de trabajo. Así lo evidencian los estudios de mercado antes mencionados, que identifican a la mayoría de los consumidores en el rango de edad a partir de los 25 ańos, coincidiendo con la edad promedio de inserción laboral estable (Ruiz y Castañeda, 2017).

\section{Referencias}

Aguilar, L. (2017). Mujeres jefas de hogar y algunas características de los hogares que dirigen. Una visión sociodemográfica. En Consejo Nacional de la Población (Ed.), La situación demográfica de México 2016 (pp. 109-129). Distrito Federal, México: Consejo Nacional de la Población.

Bourdieu, P. (1998). La distinción. Criterios y bases sociales del gusto. Madrid: Taurus.

Bourdieu, P. (2001). Las estructuras sociales de la economía. Buenos Aires: Manantial.

Bravo, F. y Villagra, M. (2020). Experiencia texturizada: Notas para una sociología del café de especialidad, $1^{\text {a }}$ parte. (no publicado).

Bustamante, M. y García, D. (2015). Distinction's framework. A tool to understand the space of cultural practices in Mexico. En Ph. Coulangeon y J. Duval. (Ed.), The Routledge Companion to Bourdieu's 'Distinction' (pp. 283-306). Londres, UK y Nueva York, USA: Routledge.

CAFIVER (2020). Cuatro tendencias del café en México. Recuperado de: https://blog.cafiver.com/4-tendencias-del-cafe-en-mexico/ 
Cardon, Ph. y García, D. (2012). L'alimentation: enjeux théoriques et empiriques dans les Amériques. IdeAs. L'alimentation dans les Amériques au prisme des sciences sociales 3, pp. 1-16. Recuperado de https://journals.openedition.org/ideas/403.

Consejo Mexicano Vitivinícola (2020). ¿Cuáles son los retos de la industria del vino en México? Recuperado de: https://uvayvino.org.mx/2020/03/20/cuales-son-los-retos-dela-industria-del-vino-en-mexico/

CEDRSSA (2018). El café en México, diagnóstico y perspectiva. México: Centro de Estudios para el Desarrollo Rural Sustentable y la Soberanía Alimentaria. Recuperado de: http://www.cedrssa.gob.mx/files/10/30El\%20caf\%C3\%A9\%20en\%20M\%C3\% A9xico:\%20diagn\%C3\%B3stico\%20y\%20perspectiva.pdf

De Benedittis, M. (2019). Expert Wine Tasting as a Social Practice: An Enactive Ethnography. Sociologica, 13(3), 135-147. doi: https://doi.org/10.6092/issn.1971$8853 / 9481$

Euromonitor (2017). Análisis del Mercado de Consumo de Café en México 2016. Recuperado de: https://amecafe.org.mx/wp-content/uploads/2017/08/Euromonitor_Informe_An \%C3\%A1lisis-de-consumo-2016-AMECAFE-Final.pdf.

Euromonitor (2020). Wine in Mexico. Country Report. Recuperado de: https://www.euromonitor.com/wine-in-mexico/report

Flemmen, M., Vegard, J. y Lennart, L. (2019). Class, Lifestyles and Politics: Homologies of Social Position, Taste and Political Stances. En J. Blasius, F. Lebaron, B. Le Roux y A. Schmitz (Ed.) Empirical Investigations of Social Space (pp. 155-174). New York, USA: Springer.

García, D. (2018). Le mezcal. Enfant terrible du Mexique. París, Francia: Presses Universitaires François-Rabelais.

Gennari, A. y Estrella, J. (2015). Análisis del mercado del vino en paises de Latinoamérica: México. Dolomiti, Italia: Camera di Commercio. Recuperado de https://www.tb.camcom.gov.it/uploads/CCIAA/Corsi/Atti/2015_06_23/Market_Ana lysis_mex.pdf

Giddens, A. (2017). Sociology. 5a edición. Cambridge, Inglaterra: Polity Press.

Griffith, D., Zamudio, P., Cortés, R. y Cabrera, J. (2017). Losing Labor: Coffee, Migration, and Economic Change in Veracruz, Mexico. Culture, Agriculture, Food and Environment 39(1), pp. 35-42. doi: https://doi.org/10.1111/cuag.12086.

Instituto de Información Estadística y Geográfica de Jalisco (INEGI, 2018). Ocotlán. Diagnóstico municipal. Guadalajara, Jalisco, México: Gobierno del Estado de Jalisco. Recuperado https://www.iieg.gob.mx/contenido/Municipios/Ocotlan.pdf

INEGI (2018). Encuesta Nacional de Ingresos y Gastos de los Hogares (ENIGH) 2018. Recuperado de https://www.inegi.org.mx/programas/enigh/nc/2018/. 
Hernández, E. y Calonge, F. (Coords.) (2012). Diagnóstico local sobre la realidad social, económica y cultural de la violencia y la delincuencia en el municipio de Ocotlán. Jalisco, México: Universidad de Guadalajara-Ayuntamiento de Ocotlán, Jalisco.

Mauss, M. (1923-1924). Essai sur le don. Forme et raison de l'échange dans les sociétés primitives. Edición facsimilar. Recuperado de http://www.uqac.uquebec.ca/ zone30/Classiques_des_sciences_sociales/index.html

Medina, F., Martínez, J. y Ramon, J. (2014). El mercado mundial de vino y la competitividad de los países del Hemisferio Sur, 1961-2010. América Latina en la Historia Económica, 21(2), pp. 40-83. Recuperado de http://www.scielo.org.mx/ scielo.php?script=sci_arttext\&pid=S1405-22532014000200002\&lng=es\&tlng=es

Medvetz, T. y Sallaz, J. (2018). The Oxford Handbook of Pierre Bourdieu. New York, USA: Oxford University Press.

Meléndez T. y Cañez, G. M. (2009). La cocina tradicional regional como un elemento de identidad y desarrollo local: el caso de San Pedro El Saucito, Sonora, México. Estudios Sociales, 17(núm. especial), pp.181-204. Recuperado de http://ref.scielo.org/ z5fn25

Mintz, S. W. (1996). Dulzura y poder: el lugar del azúcar en la historia moderna. México: Siglo XXI.

Moingeon, B. (1993). La sociologie de Pierre Bourdieu et son apport au marketing. Recherche et Applications en Marketing, 8(2), pp. 105-123. doi: https:/doi.org/ 10.1177/076737019300800206

Morris, J. (2013). Why espresso? Explaining changes in European coffee preferences from a production of culture perspective. European Review of History: Revue européenne d'histoire, 20(5), pp. 881-901. doi: https://doi.org/10.1080/ 13507486.2013.833717

NESTLÉ (2020). What did we do when the bank called? Invented Nescafé. Recuperado de: https://www.nestle.com/aboutus/history/nestle-company-history/nescafe

Ochoa, T. (2013). Alimentación y diferenciación social: el caso de una comunidad en México. Revista Internacional de Ciencias Sociales Interdisciplinares, 2(1), pp. 9-20. Recuperado de http://lascienciassociales.com

PROFECO (2015). ;Por mis vinos, bohemios!! México: PROFECO. Recuperado de https://issuu.com/profeco/docs/edici_n_n_m_466_diciembre_2015/62

Rojas, E., Espinoza, A., Thomé, H., Moctezuma, S. y Cuffiac, F. (2019). Understanding consumers' perception and consumption motives towards amaranth in Mexico using the Pierre Bourdieu's theoretical concept of Habitus, Apetite, 139(1), pp. 180-188. doi: https://doi.org/10.1016/j.appet.2019.04.021

Roseberry, W. (1996). The Rise of Yuppie Coffees and the Reimagination of Class in the United States. American Anthropologist New Series, 98(4), pp. 762-775. doi: 10.2307/681884. https://www.jstor.com/stable/681884 
Sandoval, S. (2012). 2012 El valor trabajo. Apuntes para un estudio de la representación social del trabajo entre habitantes de la región Ciénega. En J. Ruiz, S. Sandoval y A. Ramírez (coords.), Región y globalización. Articulación social de los mercados laborales (pp. 19-54). Saarbrücken, Alemania: Lap Lambert

Sandoval, S. (en prensa). Las relaciones de género en las transformaciones de la economía y la cultura alimentarias en una ciudad media en México. En Ma. L. González y P. Rodríguez (coords.). Políticas públicas, trabajo femenino y presupuestos de género. Avances, retrocesos y desafios. México: IEEc-UNAM

Schwarz, O. (2013). Bending Forward, One Step Backward: On the Sociology of Tasting Techniques. Cultural Sociology, 7(4), pp. 415-430. doi: https://doi.org/ $10.1177 / 1749975513480957$

Spence, C. y Carvalho, F. (2019). The coffee drinking experience: Product extrinsic (atmospheric) influences on taste and choice. Food Quality and Preference, 80, Recuperado de https://doi.org/10.1016/j.foodqual.2019.103802

Testart, A. (2014). Pourquoi les femmes ne débouchent-elles pas les bouteilles de vin? L'Homme, 212, pp. 23-35. Recuperado de https://www.jstor.org/stable/24699624

Thamer, J. (2016). Una dieta recargada. El Fast food y la construcción del gusto, movilidad y desplazamientos simbólicos. Razón y Palabra, 20(3-94), pp. 67-80. Recuperado de http://www.revistarazonypalabra.org/index.php/ryp/article/view/689

Tissot, S. (2013). 'Anything but Soul Food . Goûts et dégoûts alimentaires chez las habitants d'un quartier gentrifié. En Ph. Coulangeon y J. Duval (Ed.). Trente ans après La Distinction de Pierre Bourdieu (pp. 141-152). París, Francia: La Découverte.

Venkatachalapathy, A. R. (2002). 'In those days there was no coffee': Coffee-drinking and middle-class culture in colonial Tamilnadu. The Indian Economic \& Social History Review, 39(2-3), 301-316. doi: https://doi.org/10.1177/001946460203900209.

Vinísfera (s.f.). Mexico Wine Market, An Increasing Opportunity. Recuperado de: http://vinisfera.com/pdf/MWM1.pdf

Warde, A. (2016). The practice of eating. Londres, Inglaterra: Polity Press. 